J.M. Alaric Smith

Geoffrey E. Rose

Orbital Clinic

Moorfields Eye Hospital

London EC1V 2PD, UK

\section{P.N. Plowman}

Department of Radiotherapy and Oncology

St Bartholomew's and London Hospital

London EC1A 7BE, UK

Mr Geoffrey E. Rose

Orbital Clinic

Moorfields Eye Hospital

City Road

London EC1V 2PD, UK

e-mail: geoff.rose@moorfields.nhs.uk

Sir,

\section{Necrotising fasciitis managed without any surgical intervention}

Necrotising fasciitis is an uncommon and severe soft tissue infection characterised by cutaneous gangrene, suppurative fasciitis and vascular thrombosis usually preceded by trauma in patients with systemic problems, such as diabetes mellitus, alcoholism and intravenous drug abuse. We present a case of periorbital necrotising fasciitis which we managed without any surgical intervention and attained a satisfactory outcome.

\section{Case report}

A 74-year-old alcoholic man with diabetes mellitus and chronic liver failure presented to the Accident and Emergency Department with a history of non-traumatic swelling of his right upper/lower eyelids, and the right side of his face, associated with severe pains over a few hours. Examination demonstrated a visual acuity of $\mathrm{CF}$ in his right eye and 6/24 in the left eye unaided with no improvement with pinholes. There was marked right periorbital oedema with a dusky grey-blue appearance of the skin with surrounding irregular erythematous border and a necrosed area on the nasal aspect of his right upper lid, with an eschar $1.0 \times 1.5 \mathrm{~cm}$ (Fig. 1). Swelling extended down the right side of his face, neck and across his nose to the left lower lid. There was right axial proptosis of the globe with decreased motility, no right afferent pupillary defect and fundoscopy revealed mild pallor of both optic discs. He had a right facial palsy and his face was markedly tender and febrile to touch. No crepitus or lymphadenopathy was found.

Given his acute history and immunocompromised state, a diagnosis of necrotising fasciitis was made. He was started on intravenous clindamycin, cefotaxime and metronidazole. CT scan showed soft tissue swelling around the right eye with no collections and normal sinuses. He had an elevated white blood cell count and blood cultures were negative, but wound culture grew group A beta-haemolytic streptococcus. The cefotaxime was then replaced with high doses of benzylpenicillin every $4 \mathrm{~h}$.

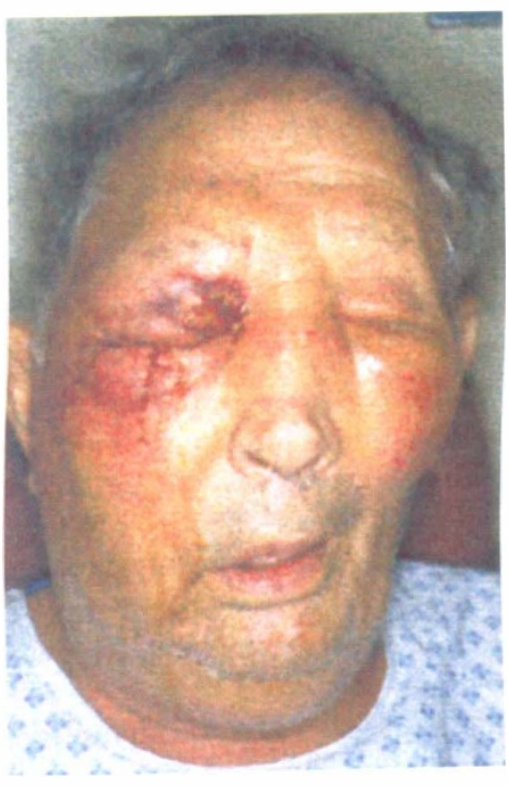

Fig. 1. Swelling of the right upper/lower lids extending down the face and to the left lower lid.

After 3 days the swelling and erythema extended to the pretracheal fascia and down the right side of his chest wall, just below the nipple line. He now had multi-organ system failure and was admitted to the Intensive Therapy Unit (ITU) for close monitoring and airway management. High doses of benzylpenicillin were maintained and his condition improved over the next 4 days when he was discharged from the ITU. The cellulitis was now resolving, but the right eye condition did not improve. The globe was now displaced laterally and downwards, with a small sinus discharging from the lateral aspect of the right lower lid. A second CT scan failed to show any collections. He was then referred to Moorfields Eye Hospital where the diagnosis was confirmed and he remained on intravenous antibiotics with no surgical debridement. The patient's condition

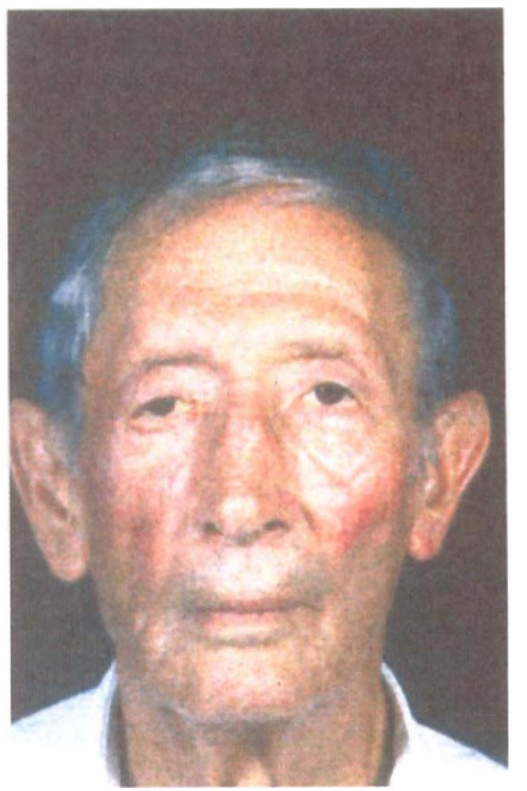

Fig. 2. Resolution 1 year later with minimal lid scarring. 
improved and he was discharged from the hospital 2 weeks later on oral antibiotics. After 1 year he has a residual partial right-sided facial palsy maintaining adequate closure of his right eye showing no signs of exposure keratopathy (Fig. 2). His corrected right visual acuity is $6 / 18$.

\section{Comment}

Necrotising fasciitis is a severe and potentially fatal soft tissue infection. The skin is initially pale red, tensely swollen and accompanied by severe pain, as necrosis spreads in the tissue planes. All authors recommend prompt diagnosis along with early surgical debridement of necrotic tissue and appropriate parenteral antibiotics. Kronish and McLeish ${ }^{1}$ even recommend hyperbaric oxygen therapy.

Although the diagnosis was made on presentation, no surgical debridement or skin grafting was performed at any time, which is usually associated with a poor prognosis. The avascularity of the fascial planes and necrotic tissue do not allow penetration of the antibiotics and, therefore, logically these areas should be debrided. However, the periorbital area is highly vascular and maintains adequate perfusion up to the lid margins. One may suggest that due to limited necrosis and very early presentation with prompt diagnosis and institution of appropriate high-dose antibiotics, adequate tissue levels were achieved early to eventually bring the infection under control. Arterial arcades of the eyelids that lie deep to the pretarsal orbicularis muscle remained unaffected, resulting in adequate perfusion of the lid margins, and the orbicularis muscle remained intact.

This patient was closely monitored throughout his acute period of illness and surgical debridement would have been performed if his condition continued to deteriorate. This report is not intended to dispute the fact that 'early surgical debridement is the mainstay of treatment', but merely to outline the importance of early diagnosis of this condition with institution of appropriate antibiotic treatment. Clindamycin has been shown to be more effective than penicillin $G$ against group $A$ streptococcus. This was our first choice in the broadspectrum cover. Clindamycin possibly controlled the infection initially, thus avoiding muscle involvement/ surgical debridement.

\section{References}

1. Kronish JW, McLeish WM. Eyelid necrosis and periorbital necrotising fasciitis. Ophthalmology 1991;98:92-8.

2. Knudson KJ, Gigantelli JW. Necrotising fasciitis of the eyelids and orbit. Arch Ophthalmol 1998;116:1548.

3. Marshall DH, Jordan DR, Gilberg SM, Harvey J, Arthurs BP, Nerad JA. Periocular necrotising fasciitis: a review of five cases. Ophthalmology 1997;104:1857-62.

4. Jordan DR, Mawn L, Marshall DH. Necrotising fasciitis caused by group A streptococcus infection after laser blepharoplasty. Am J Ophthalmol 1998;125:265-6.

5. Ray AM, Bressler K, Davis RE, Gallo JF, Patete ML. Cervicofacial necrotising fasciitis: a devastating complication of blepharoplasty. Arch Otolaryngol Head Neck Surg 1997;123:633-6.
6. Huang JW, Fang CT, Hung KY, Hsueh PR, Chang SC, Tsai TJ. Necrotising fasciitis caused by Serratia marcescens in two patients receiving corticosteroid therapy. J Formos Med Assoc 1999;98:851-4.

7. Urschel JD. Necrotising soft tissue infections. Postgrad Med J 1999;75:645-9.

8. Krohel GB, Krass HR, Winnick J. Orbital abscess: presentation, diagnosis, therapy and sequelae. Ophthalmology 1982;89:492-8.

\section{Aldrin Khan}

Nisar Ahmad

Essex County Hospital

Lexden Road

Colchester CO3 3NB, UK

\section{Sir,}

\section{Alveolar orbital rhabdomyosarcoma in a patient with} neurofibromatosis type 1

Patients with neurofibromatosis type 1 (NF1) have an increased risk of developing malignancy, usually as transformation of a neurofibroma or other tissue of neural crest origin. ${ }^{1}$ Soft tissue sarcomas, including rhabdomyosarcomas, also occur, usually in the urogenital system. ${ }^{1,2}$ We highlight rhabdomyosarcoma in the differential diagnosis of an eyelid mass in patients with NF1.

\section{Case report}

A 6-year-old girl presented with a 3 month history of a painless lump in the upper left eyelid. She had NF1, of which there was a strong family history. Visual acuity was $20 / 20$ bilaterally. A mobile, bilobed, rubbery lump was palpated in the medial left upper lid. She had ptosis, congested forniceal conjunctival vessels, and a Lisch nodule on her right iris. Ten days later the mass had increased markedly in size (Fig. 1). An MRI scan showed a large non-enhancing pre-septal mass extending superiorly to the orbital margin, indistinguishable from overlying soft tissues, with lateral extension into the lacrimal region.

Subtotal excision was performed and histological examination showed an alveolar-type rhabdomyosarcoma, confirmed by the regional paediatric pathology service and United Kingdom

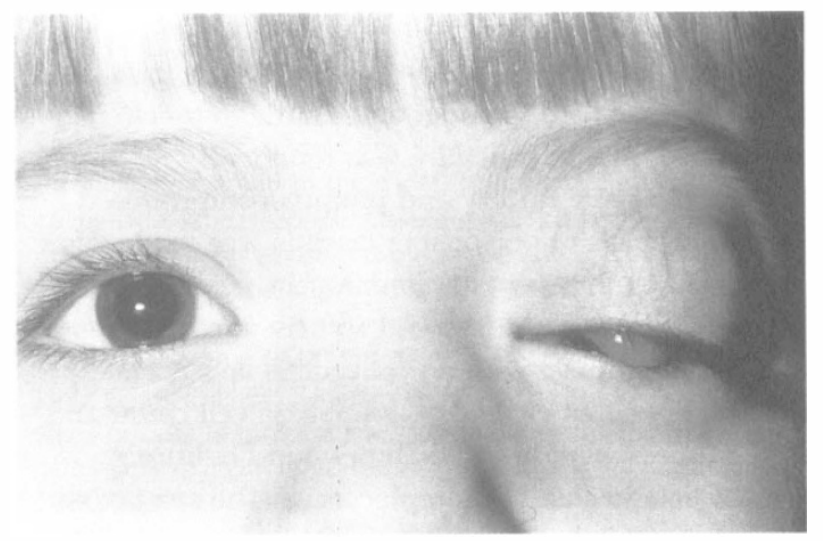

Fig. 1. The appearance before treatment. 